\title{
Filigrane
}

Écoutes psychothérapiques

\section{Le travail du temps dans la cure psychanalytique : le corps-analyste comme kolossos}

\section{Céline Masson}

Volume 17, numéro 1, printemps 2008

L'avenir du clinicien I

URI : https://id.erudit.org/iderudit/018789ar

DOI : https://doi.org/10.7202/018789ar

Aller au sommaire du numéro

Éditeur(s)

Revue Santé mentale au Québec

ISSN

1192-1412 (imprimé)

1911-4656 (numérique)

Découvrir la revue

Citer cet article

Masson, C. (2008). Le travail du temps dans la cure psychanalytique : le corps-analyste comme kolossos. Filigrane, 17(1), 51-67.

https://doi.org/10.7202/018789ar
Résumé de l'article

Dans cet article, il s'agira d'interroger le corps dans la séance d'analyse et ce qu'il implique comme dimension temporelle. Cette création d'un lieu analytique est aussi l'advenue d'un corps de présence, un corps s'incarnant dans le lieu de mémoire qu'inaugure le lieu de l'analyse. Le lieu pour un temps et un corps dans l'espace redessine le corps des pulsions et c'est bien dans la parole que toutes les dimensions temporelles se conjuguent au présent, là dans les séances et dans leur entre-deux. Lorsque des images surgissent dans le transfert, c'est une pulsation qui apparaît, un mouvement pulsionnel qui réactive ces images dans le temps. Les images fixes sont des images qui sidèrent, rendent difficiles la venue d'autres images et empêchent le mouvement de la pensée. Les images en mouvement créent du lieu en recréant du lien et l'analyste est ce corps en présence qui permet la dé-sidération et la relance du désir par l'installation d'un cadre (temps et lieu). Dans ce mouvement de va-et-vient de la présence-analyste, le sujet fait l'épreuve de la perte et du manque, de l'absence et de la présence d'un corps-aliment. 


\title{
Le travail du temps dans la cure psychanalytique: le corps-analyste comme kolossos
}

\author{
céline masson
}

Dans cet article, il s'agira d'interroger le corps dans la séance d'analyse et ce qu'il implique comme dimension temporelle. Cette création d'un lieu analytique est aussi l'advenue d'un corps de présence, un corps s'incarnant dans le lieu de mémoire qu'inaugure le lieu de l'analyse. Le lieu pour un temps et un corps dans l'espace redessine le corps des pulsions et c'est bien dans la parole que toutes les dimensions temporelles se conjuguent au présent, là dans les séances et dans leur entre-deux. Lorsque des images surgissent dans le transfert, c'est une pulsation qui apparaît, un mouvement pulsionnel qui réactive ces images dans le temps. Les images fixes sont des images qui sidèrent, rendent difficiles la venue d'autres images et empêchent le mouvement de la pensée. Les images en mouvement créent du lieu en recréant du lien et l'analyste est ce corps en présence qui permet la dé-sidération et la relance du désir par l'installation d'un cadre (temps et lieu). Dans ce mouvement de va-et-vient de la présence-analyste, le sujet fait l'épreuve de la perte et du manque, de l'absence et de la présence d'un corps-aliment.

\begin{abstract}
«Lorsqu'on voit tant de pierres sépulcrales enfoncées en terre, ou bien usées sous les pas des fidèles, et les églises mêmes écroulées sur leurs tombeaux, on peut toujours se représenter la vie après la mort comme une seconde vie, dans laquelle on entre par une image, par une inscription, et dans laquelle on subsiste plus longtemps que dans la véritable vie des vivants; mais cette image, cette seconde existence, s'évanouit tôt ou tard. Le temps ne se laisse pas ravir des droits sur les monuments plus que sur les hommes.» (Goethe, 1995, 221)
\end{abstract}

\footnotetext{
'est le texte de Pierre Fédida, «L'interlocuteur» (Fédida, 1995, 121-186) qui m'a permis de poser cette hypothèse que la personne du transfert qu'est l'analyste pourrait être un équivalent de kolossos tel que Jean-Pierre Vernant le définit (Vernant, 1990). Fédida reprend la notion de totem à partir de l'étude de Freud, Totem et tabou, et dit:
}

«Cette démonstration [dans le livre de Freud] accorde, en effet, au totem la fonction d'imaginer (plutôt que représenter) l'ancêtre mort et d'attendre de celui-ci une filiation non-par-lesang, assurant protection et identité psychique de l'individu sous réserve que ne soient pas transgressés les tabous qui s'y rattachent. La consanguinité de ceux qui sont issus du même 
totem fonde non seulement l'interdiction du commerce sexuel entre les mêmes mais positivement l'idée de parenté totémique et de transmission transférentielle.» (Fédida, 1995, 172).

On se souvient de la saisissante proposition de Freud sur l'origine du totémisme telle qu'il a pu la reconstituer:

«Un jour, les frères qui avaient été chassés se coalisèrent tuèrent et mangèrent le père, mettant ainsi fin à la horde paternelle. Unis, ils osèrent entreprendre et réaliser ce qu'il leur aurait été impossible de faire isolément [...] qu'ils aient également mangé le cadavre va de soi pour le sauvage cannibale. Le père originaire tyrannique avait certainement été le modèle envié et redouté de chacun des membres de la troupe des frères. Dès lors, dans l'acte de manger, ils parvenaient à réaliser l'identification avec lui, s'appropriaient chacun une partie de sa force $[\ldots]$ » (Freud, 1993, 290).

Le totem est désigné par Freud comme substitut du père (son effigie) qui, comme le dit bien Fédida, porte témoignage du meurtre et de son reniement. Mais ce qui demeure, ce n'est pas tant la figure que le nom ou l'écriture du nom, le bien écrit totem. Et Fédida de nous mettre sur la piste du transfert totémique:

«Il serait fort tentant de conclure à une sorte de nature totémique du transfert [...]» (Fédida, 1995, 173).

Survivance névrotique du totem dans le lien religieux. Fédida avance cette hypothèse afin d'interroger la continuité du psychique d'une génération à l'autre ainsi que la question essentielle du généalogique dans le transfert.

«Comme s'il fallait alors cette personne totémique bien sûr comme "substitut du père", mais surtout comme ce qui, dans un substitut, ne désigne pas un représentant ou un "tenant-lieu" mais plutôt l'apparence d'image ou la semblance qui appartient à l'absence du père, c'est-à-dire sous laquelle cette absence est le foyer d'illusion de sa présence et existe comme vide inimaginable.» (Fédida, 1995, 174).

«Personne totémique», «substitut», «semblance», «absence du père» sont des expressions qui m'ont ramenée au kolossos. Quelque chose évoque le disparu, une présence massive (de la pierre) qui ne représente pas mais se contente d'évoquer, de rappeler par certains traits qui ne sont pas forcément physiques, mais des traits de présence. Telles sont les caractéristiques de l'analyste, d'une 
présence en traits. Le totem-analyste, tel un kolossos, fixerait le fantôme par l'écriture du nom qu'il permet dans ce travail du langage. La pierre est ici la présence des mots de la langue qui viennent en lieu et place dans le temps de l'analyse, en la demeure de l'analyste. Enfin, les mots deviennent la sépulture des morts en errance qui n'ont cessé de perturber l'imaginaire des sujets, ils étaient bien trop imaginables. Ils sont désormais fixés dans les mots entendus par l'écoute de l'analyste. Les morts sont mots là où les mots étaient morts enlisés dans la présence des morts qui ne voulaient pas trépasser. Quand on est envahi par l'imaginaire on ne peut plus parler, les images sidérantes captent alors les mots de la langue. Le processus transférentiel permet des changements de lieux qui sont des changements dans la temporalité langagière et psychique. La parole (référée à cette présence de l'analyste) fait déplacer le lieu depuis ce «passé immémorial» (Fédida), là où résident les figures des ancêtres. C'est le «présent des mots » qui actualise une personne, un interlocuteur, et c'est par cette massification de la présence (qui fixe) que le travail de langage est possible. C'est de la mémoire du temps que l'on peut faire surgir un kolossos comme présence libérant la parole.

«Temporalités paradoxales du transfert si l'on n'oublie pas de même que - tout comme pour le rêve — les personnes sont des apparences des morts. Travail du deuil que serait le transfert? Peut-être si l'on entend toutefois ce retour des morts depuis leur deuil impossible, à la recherche de leur sépulture dans le nom qui les nomme. » (Fédida, 1995, 180).

La voie associative de la parole rendue possible au lieu même de l'analyse restitue aux mots le pouvoir réminiscent des images qu'ils contiennent. Et la parole en présence retrouve les métaphores langagières et la mémoire des lieux c'est-à-dire aussi la mémoire des langues. C'est lorsque les lieux d'histoire et de mémoire sont en place que le pathos visuel peut être réactivé. Remettre les lieux en place dans la mémoire, c'est aussi redonner une place aux morts, aux « disparus de l'œil», si je peux me permettre cette expression, ceux que l'on a laissé dans l'histoire, ceux qui ont fait notre histoire. Si les places ne sont pas claires (et je joue volontairement sur des métaphores visuelles), les fantômes continueront de hanter le sujet.

La parole devient le lieu de la remémoration, de cet état de Hilflosigkeit (état de détresse), cet hors-langage qu'elle tente de cerner pour rendre ce moment de détresse plus supportable dans son évocation.

Image du passé qui s'évanouit avec chaque présent qui n'a pas su se reconnaître visé par cette image du passé. N'est-ce pas là aussi qu'opère le travail de la cure ? Dans ce ressaisissement du passé par le transfert qui est justement visé par cette «image unique, irremplaçable du passé ». Le transfert, ce moment présent, de visà-vis, est concerné par ces images uniques, il se reconnaît peut-on dire avec Benjamin, visé par elles... Il dira plus loin que: 
«Le "présent", modèle des temps messianiques, ramassant, tel un raccourci formidable, en soi l'histoire de l'humanité entière, correspond très exactement à la place qu'occupe cette histoire au sein de l'univers. » (Benjamin, 1991, 443)

Le «présent» du transfert «ramasse» dans un raccourci, l'histoire du sujet voire même sa pré-histoire. La pensée benjaminienne du temps nous éclaire encore, par un autre point de vue que le point de vue freudien, sur les processus psychiques et notamment le travail du temps psychique.

Afin d'illustrer ce qui vient d'être dit, j'aimerais reprendre cette question de la figure de l'analyste à partir d'un rêve d'une patiente qui est en analyse depuis plus d'un an.

«Elle rêve qu'elle avait rendez-vous chez la psychologue mais qu'elle avait oublié car c'était les vacances et qu'elle avait beaucoup de choses à faire. Elle devait lui téléphoner mais ça la gênait, elle s'y rendit directement. Au fur et à mesure que je parlais, elle ne m'écoutait plus, elle s'est levée, je la suivais comme si c'était une l'école. Quelque chose était écrit sur sa tête. Son attitude signifiait qu'elle ne voulait plus m'écouter. J'avais la volonté de continuer, elle s'est retournée vers moi et me dit: "Vous ne me parlez pas de ce qui est important". Elle s'est approchée de moi et m'a demandé de sortir ce qui était important. »

La veille du rêve, elle a regardé une émission sur Jean-Paul II, ce qui l'a renvoie à la confession. Elle-même est croyante. Elle associe sur le fait qu'elle se trouve dans une situation où elle doit faire quelque chose qui lui est imposé par les autres. Elle projette sur la figure de la psychologue, deux personnages, à la fois sa mère qui lui impose ses désirs et une maîtresse qui a été autoritaire avec elle. On voit comment la figure du «psy» vient «ramasser»d'autres figures importantes et notamment sa mère qui représente pour elle une personne changeante, qu'elle craignait et craint toujours (même si le travail qu'elle fait lui permet de prendre de la distance). Je n'ai pas le temps de développer plus avant ce cas et les très nombreux rêves que cette patiente rapporte à chaque séance mais il est sûr que cet espace de parole et de temps permet que se déploie une parole empêchée et inquiétée par un interdit posé à l'endroit même d'une parole vraie concernant notamment des secrets générationnels (la mère de cette patiente a été élevée par ses grands-parents comme d'autres membres de la famille et notre patiente en ignore la raison... elle parle d'enfants délaissés). Ce qu'elle travaille en séance, c'est le mode de relation qu'elle a créé à sa mère et qui la terrifie parfois. Une mère-maîtresse qui dicte ses lois et dont la relation a servi de mode de relation assujettissant aux autres. Comme elle dit, elle se soumet aux désirs des autres. 
L'analyste représente par déplacement celui à qui tout dire signifie injonction de dire, dire pour plaire à l'autre et en être gratifié. Mais il y a aussi la crainte chez cette patiente que l'analyste «change», passant d'une position d'écoutante bienveillante à celle de rejet. Je réitère la phrase que je disais plus haut que le "présent» du transfert «ramasse » dans un raccourci, l'histoire du sujet voire même sa pré-histoire.

\section{La «magie lente» et le temps infini}

«[...] ils parlent ensemble» (Freud, 1926, 33). On se souvient de cette réponse de Freud à l'interlocuteur impartial qui se demandait ce qui se passe entre un analyste et son patient (La question de l'analyse profane). Est-ce donc une sorte de «magie» se demande encore cet interlocuteur imaginaire ? Ce serait de la magie si cela agissait avec la rapidité de l'éclair, en un rien de temps. Or le temps nécessaire, indispensable au déploiement de la parole fait perdre «le caractère du merveilleux » propre à la magie. C'est le temps dans la langue qui est porteur, à l'insu du sujet parlant, des origines, de l'histoire, des formes transmises de génération en génération. Les mots de la langue viennent dans le temps de la parole, dans la voix haute adressée à l'autre du transfert. La cure est un travail de la langue à l'épreuve du temps, un travail du temps dans la langue même, celle du dessous, la plus proche du corps. La magie opère au travers de cette parole agissante dans le temps et de la circulation des mots. «Magie lente» (P. Lacoste) où l'un et l'autre des présents s'inventent une langue propre au transfert, une petite langue intime et étrangère. Entendre les bruits de l'inconscient que cette langue, la petite du fond, ramène dans la parole de temps, dans la parole d'histoire au présent. Comme le dit justement Freud dans le Moïse, les mots sont traités comme des précipités psychiques verbaux, des précipités de temps.

«Ce "tôt ou tard" contient une allusion à l'importance du facteur temps, pour qu'une analyse puisse être entièrement terminée. Ceci n'est possible que si l'analyse dispose d'un temps, pour ainsi dire, infini. Je suis donc d'accord avec ceux qui prétendent qu'un traitement a d'autant plus de chances d'aboutir rapidement que le temps dont nous disposons est illimité. Il s'agit ici moins du temps physique dont le patient dispose, que de sa détermination intérieure de tenir vraiment aussi longtemps qu'il sera nécessaire, sans égard pour la dimension absolue du temps. » (Ferenczi, 1974, 48).

Ces propos de Ferenczi souligne l'importance du temps nécessaire à l'élaboration du matériel psychique inconscient (Durcharbeiten, perlaboration ou translaboration suivant les traducteurs). La question du temps traverse toute l'œuvre freudienne même si elle ne fait pas l'objet d'une élaboration métapsychologique: organisation libidinale, régression, répétition, souvenirs et 
réminiscences, Nachträglichkeit (après-coup). Ce dernier terme est utilisé par Freud en relation avec sa conception de la temporalité et de la causalité psychiques. Freud a montré que le sujet remanie après-coup les événements passés et c'est bien cette reconsidération qui leur donne un sens voire même une efficacité ou une action négative. L'après-coup enfile dans une même figure de temps à la fois l'événement efficace qui provoquera une formation symptomatique actualisant dans un maintenant, le traumatisme (par l'irruption d'une ou d'un ensemble de représentations), ce qui suppose que l'événement traumatisant demeure en état de veille dans le psychisme. Ce mode de survivance de l'événement, non actuel, engendre une temporalité à plusieurs voies puisque c'est l'irruption d'un nouvel événement qui anime le premier. Après-coup, l'événement prend ses marques dans le corps là où il n'avait qu'effleuré la surface psychique. Collision de deux présents séparés dans la durée mais rendus actuels dans le temps psychique. La représentation en soi banale va accueillir, dans un espace-temps commun, les traits d'un événement suspendu dans le temps. C'est bien dans cet espace-temps que s'inscrit le fonctionnement psychique.

La représentation du temps naît de la perception d'une rythmicité interne du fonctionnement de l'appareil psychique, un mouvement établissant un rapport entre le dedans des formes et le dehors des choses. Le temps psychique est d'abord un temps perceptif sensori-moteur, affectif et pulsionnel (les pulsions étant à la base de la vie affective, elles sont ressenties sous la forme de désirs comme des forces venant de l'organisme vers un objet pour se satisfaire. Elles ne tiennent tout d'abord pas compte de la réalité puis s'y intègrent). Cette construction du temps s'élabore à partir du moment où dedans et dehors se dialectisent en instaurant une motricité qui fait aller le sujet dans le monde et vers les autres. C'est cette tension moi-l'autre, sujet-objet qui installe une temporalité interne nécessaire à la conscience du temps. Temps psychique et temps réel entrent alors en coïncidence. La pulsation du temps se fait autour d'une figure inconnue, tendue vers une absence. Les impressions reçues subsistent non seulement, dit Freud, telles qu'elles ont été reçues quant à leur nature mais en conservant les formes qu'elles ont prises au cours de leur développement. Le travail des formes dans le psychisme engendre une construction mnésique qui est la concrétion figurale des états successifs des impressions reçues et de leur progressive transformation. La mémoire est ainsi ce monument d'histoire qui a résisté au temps et qui ne cesse de le déjouer.

\section{Les formes de temps dans la cure: créer du temps}

La préhistoire des névroses nous l'enseigne: l'enfant a recours à cette expérience phylogénique là où son expérience personnelle ne suffit plus. Il comble les lacunes de la vérité individuelle avec la vérité préhistorique, il remplace sa propre expérience par celle de ses ancêtres. (Freud, 1990, 399-400)

Le temps dans la cure est visible, perceptible. Nous voyons des formes de temps qui s'inscrivent au fil des séances dans l'espace de paroles. Et de nouvelles formes 
de temps s'y inscrivent à la surprise parfois des patients. Comme en musique, le temps s'écrit sur des portées qui se jouent. Rythmes, pulsations, altérations, silences, signes, voix, mouvements, modes majeur/mineur, ornements, abréviations, expressions, accords, cadences, modulation, formes, tonalités, répétitions, motifs. Autant de variations traduisant des états psychiques nuancés. Nous voyons un temps se jouer avec autant de variations différentes. Cette pensée me conduit à dire que la musique c'est du temps et que finalement le travail de la parole dans la cure est un temps éminemment musical (des auteurs comme Alain Didier-Weill en a déjà remarquablement parlé). Ces notes de temps inédites étaient à jouer maintenant, car c'était en ce lieu que la partition se rendait visible. C'est un peu comme parler une langue qu' on croyait ignorer et qu' on se met un jour, en ouvrant la bouche, à en émettre des sons : «je me suis mise un jour à parler yiddish alors que je croyais ne pas connaître cette langue» me disait une personne proche. Les notes sont inscrites mais ce sont les conditions (cadre, lieu, moment dans l'histoire) de leur mise en jeu qui sont nouvelles et inédites. Alors nous jouons des gammes tout à la fois familières et nouvelles et cette mise en résonance contribue à créer du temps. J'entends par là un temps audible et adressé, un temps composé de nuances et ouvert sur l'avant, un temps sans bornes, un temps plastique généré par ce dispositif original. Ce temps joué rend possible le passage des souvenirs encore non advenus et leur mise en mots. Ce temps joué (qui n'est pas le temps de la séance) installe l'expérience analytique, en ce sens qu'il permet à la voix intérieure d'être audible. Il a des rythmes propres qui permettent des mélodies variées et contribue au dégel des représentations. Le temps dans la cure, tel un sculpteur, travaille la matière psychique, dégrossit une matière brute, atonale afin de dégager la forme qu'elle contient, son essence même. Taille et formation d'une figure, passage de l'informe à la mise en forme, création d'un espace et d'un lieu pour les événements psychiques parfois anachroniques. C'est bien ce travail du temps qui permet de donner une matière au son, de refaire glisser du sens sous les signifiants afin que le son devienne musicalité et donc écoute, une attention portée aux sons. C'est le corps qui sera le lieu de la résonance tel un instrument, prêt à accueillir le nouvel équilibre sonore, la nouvelle matérialité des sons adressés. Le silence dans les séances est également ce corps résonnant, un lieu de l'écho des sons de l'inconscient. Ce silence est organisateur de l'espace/temps qui rendra possible la reconnaissance du familier, des voix familières qui portent haut cette fois dans le temps de la rencontre. Il ressuscite un temps affectif en créant les conditions de son souvenir là précisément où les images vont toucher aux sons et refaire sens, ce qui permettra une déprise affective et une reconfiguration harmonique des affects. C'est bien dans l'espace-temps pris dans l'écoute que se reconstitue du sens à partir du son, un son espacé en quelque sorte. Cet espace clos et à la fois infini dans ses possibles, découvre la toute puissance des sonorités et règle le rapport de la présence à l'absence. Dans cet entre-deux surgissent fantasmes et fantômes de temps dans leur toute puissance visible cette fois-ci. «C'est en vous parlant que je réalise...» entend-on souvent énoncer les patients. «Réaliser», matérialiser, se 
saisir de l'informe et lui donner un contour est rendu possible dans cet espace du silence qui compose un milieu sonore capable de rendre à l'écoute sa dimension inconsciente. C'est à partir de cette musicalité à l'œuvre que l'analyste est en mesure de relever l'organisation mythique primitive qui la sous-tend.

\section{Des figures de parole}

La cause de notre douleur, comme de notre joie, est ainsi le plus souvent hors du présent, de l'actuel; elle réside dans les pensées tout abstraites; ce sont elles, ces pensées, qui souvent nous accablent de leur poids et nous infligent ces tortures [...]. (Schopenhauer, 1989, 379)

Le travail de la cure consiste à faire émerger des figures par le pouvoir de la parole afin de reconstruire des lieux du corps. Un corps ayant parfois perdu sa forme, laissé visible sans contours à la recherche désespérée d'une surface de réflexion («dis-moi qui je suis car je n'ai pas d'image, je suis laissé sans forme visible dans le monde. Me voit-on?» Tels pourraient être les propos de nombres de patients affectés narcissiquement dans leur image et leur sentiment d'être soi. Ferenczi parle de «l'anéantissement du sentiment de soi» en raison d'une «commotion psychique»(Erschütterung)). Cet anéantissement de ce «sentiment de soi » est aussi perte d'une temporalité interne qui contribue au maintien de cette forme. Le travail analytique qui est travail des formes psychiques (comme le travail du rêve) engendre des surfaces qui sont autant de points d'ancrage pour recomposer ce corps de lettres (corps de jouissance). Le travail par la parole c'està-dire une route entreprise d'accès à son propre inconscient (terre aussi étrangère au sujet qu'une terre extérieure) permet la délivrance de son propre langage — une mise en contact de la langue du dessus (langue parlée effectivement, langue dite maternelle) avec la langue du dessous (langue gardée, conservée - langue des Mères). Cette mise en contact des langues par la parole rend possible l'émergence d'affects insoupçonnés et l'accès à un langage propre.

Cet accès est fondateur d'un Lieu - lieu de type onirique susceptible de reformer un corps (corps de jouissance-corps de lettres) d'une nouvelle manière. Freud parlait de «magie des mots sur le corps», mots qui activent ainsi une mémoire de la sensorialité. Un lieu ainsi ouvert permet l'activation d'une temporalité et la venue des figures. Ces figures présentes/absentes se visibilisent à partir du moment où la parole s'exerce. L'invisible qui hante l'image, c'est la parole. Il s'agit de tirer au clair — rendre lisible par la visibilité d'une scène - c'est ce qu'ont fait les grecs. L'art de la tragédie consiste à tirer des ténèbres les affects pour les mener sur la scène sociale du partage — espace civique du partage de la langue dont le théâtre en est le paradigme. Le lieu signifie le Lieu de la parole et du regard communs. La création d'un Lieu pour la parole et les affects créent aussi les conditions pour que certaines inscriptions mnésiques s'accomplissent et rendent possible la retrouvaille avec les signifiants. Si les inscriptions mnésiques ne trouvent pas ce lieu pour l'élaboration psychique, il y a de fortes chances pour 
que les expériences douloureuses (de type traumatique) ne soient pas métabolisables autrement dit le sujet ne pourra investir ce qui advient. Le sujet est suspendu dans une confusion des corps donc dans une confusion temporelle, ramené à l'identique de l'identique dans une impossibilité de projection et récusant toute différence.

S'il n'y a pas de lieu pour le psychique il ne peut y avoir de temps pour la pensée et la créativité. Le sujet est capturé dans un non-sens existentiel saisi dans un ni-vie-ni-mort stagnant dans des eaux troubles, asséchant ainsi toute vie psychique et affective. Le temps travaille au corps le sujet sans quoi ce corps est sans limite (corps émietté, en morceaux). Ce qui arrive ne trouve pas de lieu d'inscription et le sujet est sans rythme intérieur entièrement dépendant des flux externes. Univers sans médiation dans un collage avec l'instant (le réel immédiat) annulant les limites et les espaces. Véritable angle mort où le sujet, ne disposant d'espace visible, ne se voit pas venir. Dans le travail engagé dans la cure, le sujet est face à un autre qui le regarde et entend créant ainsi une véritable surface spéculaire pour l'inscription des traces témoignant d'un ici-maintenant-vivant. Ce processus est décisif pour la reconduction d'une temporalité psychique et l'ouverture des zones d'ombre (l'inconnu en soi). Ce lieu et ce lien (transférentiel) permettent l'émergence de métaphores, de figures de temps. Lorsqu'un patient mobilise sa parole, il saisit en même temps des temps de niveaux différents qui amènent aussi des images (re)composées. Le langage est traversé par le temps et contribue aussi à une énonciation singulière où se croisent des fragments hétérogènes de temps. L'analyste est un accélérateur d'images, de fantasmes rendant possible un contact avec l'infantile, un temps anachronique de la mémoire en survivance. Ces mouvements (de temps, répétons-le) introduisent des rythmes et des modes d'être à l'autre singuliers et contribuent à ouvrir les voies d'accès à ce lieu de l'infantile. Ce lieu du temps par excellence, c'est-à-dire des formes possibles de temps.

L'analyse met en activité ces possibles en créant pour chaque sujet son lieu, un lieu d'entrecroisement des formes de temps. Un temps qui redonne de la présence, un processus d'humanisation là où l'informe et la désorganisation régnaient. L'inaperçu fait retour dans le flot de paroles qui éclaire des événements insignifiants. L'événement apparaît alors non pas comme fait passé mais comme fait actuel créant un nouveau moment de temps. Ce nouveau moment de temps porte la trace d'une autre expérience qui s'est portée à la mémoire et qui a traversé l'histoire comme un fantôme. Cet éclat, ce symptôme de temps qui se visibilise dans l'image présente est, comme dit Walter Benjamin, «collision du maintenant et de l'autrefois ». Ce brusque choc crée une nouvelle image, un accident de temps qui fait irruption sur la surface psychique. Choc entre deux événements hétérogènes séparés par une infinité de secondes et pourtant si présents l'un à l'autre, si proches l'un de l'autre. Cette co-présence crée du lieu mais un lieu d'entre-deux où d'autres réalités vont s'inscrire. Un lieu ouvert de temps où les images sont rendues possibles par cette brusque ouverture des représentations. Cette création de lieu est aussi l'advenue d'un corps de présence, un corps s'incarnant dans le 
lieu de mémoire qu'inaugure le lieu analytique. Le lieu pour un temps et un corps dans l'espace redessine le corps des pulsions et c'est bien dans la parole que toutes les dimensions temporelles se conjuguent au présent, là dans les séances et dans leur entre-deux. Le corps reprend ses différentes temporalités dans le langage. Dès 1896, Freud, dans sa Lettre 52 à Fliess, remarquait que notre mécanisme psychique était composé de couches successives et s'était construit par un processus de stratification (Freud, 1986, 153-160) ${ }^{1}$. Différents enregistrements et inscriptions se produisent et laissent des traces (dites mnésiques) qui s'ordonnent dans un réseau associatif. C'est ce qui fait dire à Freud dans la même lettre que la mémoire est plusieurs fois présente et qu'elle se compose de diverses sortes de signes. Cette mémoire est plastique, modifiable et remaniable, comprenant des espaces différents et une hétérogénéité de lieux d'inscription. Quand un événement se produit et que sa charge perceptive et affectale percute la surface de la mémoire, il se produit un remaniement de cette dernière et une forme nouvelle de temps surgit.

Freud, dans son texte le «Bloc-notes magique» (Freud, 1985, 119-124) ${ }^{2}$ topologise une mémoire plastique et remaniable en fonction des nouvelles traces qui s'inscrivent. Ce temps ainsi formé, qui ignore la durée, dispose d'événements séparés dans leur durée mais proche dans leur contenu affectal, ce qui les fait ressurgir dans la simultanéité du travail des associations libres. Les différentes traces mnésiques sont tissées ensemble et se rendent présentes ensemble dans le temps du ressouvenir et c'est bien ce devenir ensemble des traces qui historicise les événements qui ont eu lieu dans un passé et que le sujet se réapproprie. Cette collision du maintenant et de l'autrefois ré-ouvre un lieu et permet des passages d'images et de représentations. Une «bousculade» heureuse d'événements de temps se produit, événements qui avaient été refoulés et qui se trouvent convoqués et inscrits dans le langage. C'est l'événement nouveau qui permet le redéploiement de l'ancien dans la présence. Cet ancien était là mais désactivé et désaffecté, maintenu en raison de forces pulsionnelles agissantes. C'est cette expérience temporelle qui va permettre la construction du lieu. Le temps est un préalable nécessaire pour construire un espace (psychique), un espace pour les pensées. Il est un temps identifiant qui pulsionnalise le corps du désir. Dès lors, c'est un corps désirant (tendu vers un Autre) qui est pris dans un rapport au temps, un temps du désir (le désir du sujet parlant est le désir de l'Autre). Ce rapport à l'Autre est un rapport d'inconnu qui échappe au sujet et c'est bien ce point de fuite au cœur du temps qui rend possible la rencontre avec l'extérieur (cette relation à l'Autre est un préalable à la rencontre avec un semblable). Le temps crée du lieu mais crée aussi de la relation car il provoque la dimension de l'entre-deux, entre l'identique et la différence. Sans cette tension d'entre-deux, difficile mais nécessaire, il y a le risque du même, un sans-lieu car un lieu sans manque (là où travaille le désir). Et cette tension est ce qui va constituer le sujet parlant, qui va le rendre parlant à ne rien dire ou plutôt à dire rien car concernant le manque, il y a rien à dire. C'est par ce rien qu'il est un sujet du désir et qu'il sera confronter à ce qu'il n'est pas. 


\section{Le temps du transfert: créer du lieu}

L'instauration d'une temporalité dans le transfert s'opère à partir de «l'étrangèreté » de la relation transférentielle: un sujet qui parle pour un autre qui reçoit sa parole là où il n'est pas: sorte de point aveugle communicationnel. On ne s'y voit pas. L'analyste n'est pas le récepteur au sens des théories de la communication. La parole du locuteur-analysant ne lui est pas adressé (même si une demande d'analyse a été formulée et adressée dans un premier temps ${ }^{3}$ ) et pourtant il lui parle et le récepteur-analyste n'est pas le destinataire du message et pourtant il l'entend. Cette relation et ce dispositif vont créer une temporalité spécifique à l'analyse qui va rendre possible, à partir des réseaux associatifs qui s'y déploient aussi bien du côté analyste que du côté analysant et à partir des répétitions, l'émergence d'une langue inconnue ornée d'une mélodie à la fois familière et étrangère. C'est l'inquiétante étrangeté du transfert prenant appui sur un noyau inconnu, un ombilic au cœur même du langage, qui exerce la mise à flots d'émotions nouvelles créant alors de nouveaux rythmes. On ne s'entend pas car on n'y est pas et c'est bien cette rencontre échouée, ce ratage de la communication (du lieu où j'entends, je n'y suis pas et la parole adressée fait sans cesse retour au lieu même où c'est énoncé) qui permet l'instauration d'un temps transférentiel. Ce temps dépend éminemment de l'écoute de l'analyste, cette écoute d'un lieu Autre, une écoute altière oserions-nous dire, une écoute qui prend la parole de haut afin de rendre possible des espaces. Un contact dans l'espace, presque musical, laissant se déployer la partition sans interférer sur son tempo.

Par cette écoute nécessaire, des figures émergent, inédites et les images se relient balancées d'avant en arrière, percutant toutes sortes de perceptions nouvelles. Le temps dans la cure analytique est toujours projeté vers l'inconnu pour revenir vers le familier et l'intime, un saut dans un espace peu fréquenté drainant avec soi des restes inassimilables. C'est bien la rencontre avec cet inconnu en soi qui effraie le patient et l'incite parfois à prendre la fuite car cette rencontre est abyssale («je ne sais pas où je vais, je ne sais pas à quoi ça mène de parler comme ça dans le vide» entend-on bien souvent dans les premières séances). Ce temps transférentiel est un temps de relance imaginale, de réactivations d'images de temps coïncées dans des identifications narcissiques. Lorsque des images surgissent dans le transfert, c'est une pulsation qui apparaît, un mouvement pulsionnel qui réactive ces images dans le temps. Les images fixes sont des images qui sidèrent, rendent difficiles la venue d'autres images et empêchent le mouvement de la pensée. Les images en mouvement créent du lieu en recréant du lien et l'analyste est ce corps en présence qui permet la déprise et la désadhérence par l'installation d'un cadre (temps et lieu). Dans ce mouvement de va-et-vient de la présence-analyste, le sujet fait l'épreuve de la perte et du manque, de l'absence et de la présence d'un corps-aliment. Cette relation, singulière, est d'abord l'instauration d'une temporalité et du maniement par l'analyste de l'effet produit par la répétition de ce temps de la rencontre. Ce temps est le temps de toutes les présences qui rendent manifeste le désir. Ces présences sont la réactualisation de 
rencontres au prisme de l'actuel. En ce sens, ce lieu nouveau est un lieu pour le non-lieu des événements psychiques qui revenaient sans cesse sans espace géographique pour s'inscrire.

C'est entre l'analyste et l'analysant que se constitue ce lieu, entre la série des associations côté analysant et les interprétations côté analyste. Ainsi les événements qui arrivent font résonance car il y a un lieu pour les recevoir. La parole se réapproprie l'événement dans le lieu du transfert pour le faire devenir là où il a pris lieu, à un moment de l'histoire du sujet. En prenant la parole dans ce temps du transfert, c'est une histoire qui se redessine dans le présent réminiscent (Fédida). Prendre la parole dans le temps du transfert, c'est devenir soi dans le temps historique. C'est véritablement un processus d'historicisation et de subjectivation, un processus en mouvement jamais achevé. Voilà pourquoi nous ne pouvons pas envisager le processus transférentiel comme un processus de temps linéaire mais plutôt de temps anachronique. Ce n'est pas un passé qui se réactualise dans le présent où des conflits passés viendraient à se résoudre dans le présent du transfert mais plutôt des temps qui se «téléscopent», se «bouleversent», s' «entrechoquent» l'un l'autre afin de produire dans le transfert, des figures nouvelles. Les événements n'avaient pas eu lieu; par le transfert, ils ont lieu ici et maintenant car le temps de la parole est venu pour un sujet. Ce temps des processus inconscients, qui ne répond pas à la même logique que pour les processus conscients, est le temps de la survivance. Freud dit bien que les processus du système inconscient sont atemporels.

L'inconscient a ses temps que le conscient ne connaît pas. Les événements tels qu'ils arrivent au conscient vont être modifiés par les processus inconscients. La répétition n'est pas la reproduction à l'identique car elle a un autre lieu pour que l'événement arrive, un lieu d'écriture. C'est un moment où le destin est surpris et suspend son tracé pour laisser passer la chance de l'événement non advenu. Benjamin dit bien que le présent n'est pas le passage mais le lieu où le temps est sur le point d'arriver (Benjamin, 1983, 205-207). Il dit encore:

«L'arrêt messianique du devenir [...] est une chance révolutionnaire dans le combat pour le passé opprimé [...]. Le maintenant est le présent que le temps messianique a fait sauter et voler en éclats.» (Benjamin, 1983).

Ce «messianique » benjaminien, qui est l'effet de la rencontre avec l'Autre, est ce geste imperceptible qui fait sauter les portes du temps produisant un effet de surprise ouvrant sur un monde encore inexploré. Cet effet messianique n'est pas sans évoquer l'effet du transfert (à condition bien sûr d'en entendre la dimension anthropologique et non pas tant religieuse) où des saisissements et des contractures de temps peuvent se produire. Le transfert, c'est un effet «à l'arraché » démontant toute logique installée par le sujet. Le transfert déplace et court-circuite les catégories du temps: passé (présent ancien), présent (présent actuel) et avenir 
(futur présent). Les images venant dans le transfert conjuguent le nouveau inédit et inconnu, le passé originaire, c'est-à-dire un passé qui n'a pas eu de présent. Et c'est bien cette conjonction qui donne aux images leur force explosive et réminiscente, une force de temps psychique. Cette force se heurte aux forces venues du conscient qui elles invoquent, au nom d'un passé mythifié, un temps logique qui interdit les contradictions. Ces forces sont ce qu'on nomme les résistances.

Nous ne passons pas sur nos événements psychiques, ils restent en se modifiant dans les passages (ce sont le déplacement, la condensation, la figurabilité etc.) sous la pression des flux. Dans son texte sur «La négation», Freud écrit que:

« [...] la pensée possède la capacité de rendre à nouveau présent ce qui a été une fois perçu, par reproduction dans la représentation, sans que l'objet ait besoin d'être encore présent au-dehors. La fin première et immédiate de l'épreuve de réalité n'est donc pas de trouver dans la perception réelle un objet correspondant au représenté mais de le retrouver, de se convaincre qu'il est encore présent. [...] Mais on reconnaît comme condition pour la mise en place de l'épreuve de réalité que des objets aient été perdus qui autrefois avaient apporté une satisfaction réelle.» (Freud, 1995, 137-138).

Ce qui a été perçu revient dans l'image parce que l'objet a percuté une fois dans les yeux (appareil sensoriel visuel entre autres) et y a fait ses marques. Ce qu'il s'agit de retrouver, c'est la trace de cette empreinte de l'objet, l'image de l'objet halluciné qui apportera à nouveau la satisfaction (Lacan, 1994, 53) ${ }^{4}$.

C'est la perte et l'inscription de la perte dans le psychisme qui crée notre représentation du temps. Un temps ponctué de marques d'objets venant du dehors passant en images au dedans par les voies hallucinatoires du désir. Ce sont ces images qui entretiennent le temps présent, un temps survivant de l'objet et le fantasme inconscient en est l'organisateur. Le fantasme, image de la trace de l'objet-de-jouir, maintient la tension entre le dehors et le dedans et orchestre la vie relationnelle d'un sujet. Le fantasme est le lieu des processus ou même le processus d'un donnant-lieu qui compose avec les choses passées. Pierre Fédida cite un texte cité lui-même par G. Didi-Huberman, de Walter Benjamin (issu de «Denkbilder »):

«La langue explicite ce fait: que la mémoire n'est pas un instrument qui servirait à la reconnaissance du passé, mais qu'elle en est plutôt le médium. Elle est le médium du vécu, comme le sol est le médium dans lequel les villes antiques gisent ensevelies. Celui qui cherche à se rapprocher de son propre passé enseveli doit se comporter comme un homme qui fait des fouilles. Avant toute chose, qu'il ne s'effraie pas de revenir toujours sur la 
même et unique teneur chosale [...]. Car les teneurs chosales sont de simples strates qui ne livrent l'enjeu même de la fouille qu'au prix de la recherche la plus minutieuse. Images, qui se lèvent, détachées de tous liens anciens, telles des bijoux dans les chambres dépouillées de notre intelligence tardive, telles des torses dans la galerie du collectionneur. Il est utile, certes, pendant les fouilles, de procéder selon des plans; mais la bêche prudente et tâtonnante, elle aussi, est indispensable dans le sol sombre. Et il se leurre complètement, celui qui se contente de l'inventaire de ses découvertes sans être capable d'indiquer dans le sol actuel le lieu et la place où est conservé l'ancien. Car les véritables souvenirs ne doivent pas tant rendre compte du passé que décrire précisément le lieu où le chercheur en prit possession.» (Didi-Huberman, 1992, 128-129).

Ce qui importe est davantage le lieu actuel où se lèvent les choses passées que la teneur de celles-ci, leur contenu véritable. Car l'acte de soulever ces «images» modifie le lieu stratifié d'où elles émergent c'est-à-dire un lieu portant l'histoire de sa propre stratification. Le lieu où les «images» s'érigent tel un monument antique est un lieu actuel se présentant lors de fouilles, c'est-à-dire dans un maintenant.

Pour revenir au transfert, qu'est-il d'autre qu'une mise en condition de fouilles, l'occasion de donner un sol stratifié à ce qui vient dans la parole. Et la parole, n'est-elle pas ce lieu de réception de l'événement? Un lieu de sépulture pour le mort et un lieu vivant pour la naissance des souvenirs. Cet entrecroisement dans le transfert, de la mort et du vivant, engendre la répétition et l'actualisation du désir. Le transfert en tant que lieu et processus ouvre à la figurabilité et aux présences qui deviennent alors des figures ou mieux des sculptures, des figures taillées dans une matière informe mais une matière dure. Le lieu du transfert où passent les rêves, les images de mémoire primitive, est aussi le lieu de contact du fait de la présence de l'analyste, contact et circulation des flux d'intensité des représentations inconscientes. Le corps de l'analyste est la présence nécessaire pour que vienne ce qui n'avait pas lieu, afin justement de prendre corps. Le transfert est un lieu des visibles, des mises en présence de temporalités hétérogènes. L'analyste vient donner un tempo à la prise de parole afin que le flux de ce qui ne cesse pas de ne pas s'écrire soit lié dans la parole et le silence. La présence de l'analyste, le dispositif de la cure, sont bien des marqueurs de temps et des indications de lieux.

\section{La cure est un dispositif à relever les images}

La cure permet de relever les différents temps psychiques et de les faire résonner dans une parole, une parole qui puise au lieu du rêve. L'analyste est en lieu et place où les mots résonnent dans le temps et non sur la scène du maintenant. Maintenant est ailleurs, maintenant est pris dans une ligne de fuite temporelle, 
celle-là même qui donne son paysage à l'inconscient. Chaque mot appelle au rassemblement des temporalités sur la place de l'histoire et on voit là que la parole de l'analyste, qui marque le temps (la scansion), va véritablement soulever des événements de leurs lieux et permettre leur élaboration dans le maintenant de l'événement passé. Cette expérience recompose sans cesse le rapport à l'origine, c'est-à-dire ce point subjectif à partir de quoi une histoire est possible mais l'origine n'est ni commencement ni passé. L'origine est hors temps même si elle contribue cependant à fabriquer du temps psychique. Elle est ce point de vue à partir de quoi des modifications peuvent se produire. Et le travail de l'analyse est un accélérateur de temps qui reconfigure le point de vue (l'origine) et redispose les événements psychiques au point même de les anticiper (de les voir arriver, avoir ou prendre lieu). Cette accélération de temps est consubstantielle d'un flux d'images qui sont le support de l'événement, elles l'accompagnent tout en lui donnant un autre point de vue. Le transfert contribue donc à ce que les images soient redisposées et ce point d'origine $r e-v u$. Il est point d'appui pour la redisposition des images afin que le sujet puisse traverser les différentes identifications et reprendre place parmi les figures qui le hantent. L'analyste occupe ce site (comme dit Pierre Fédida) qui pourrait être comparé comme Freud le fait à propos de l'action connexe des deux processus du mécanisme du refoulement (Freud, 1987, 97), au sommet de la grande pyramide de Gizeh où un touriste se trouvant là sera poussé d'un côté et tiré de l'autre aussitôt.

Ce lieu de l'analyse et du transfert est un lieu où plusieurs faces travaillent en même temps, où le regard a vue sur différentes faces de temps, toutes impliquées ensemble. Fédida écrit très justement :

«Tandis que le patient recherche dans ses propres paroles l'impossible remémoration de son histoire personnelle et la levée de son amnésie, de son côté l'analyste œuvre, depuis son propre refoulement, à la construction d'une mémoire qui n'est que la construction d'une topique psychique donnant pouvoir de nommer les lieux des événements. » (Fédida, 1995, 264)

Et plus loin :

«La pratique de la psychanalyse c'est cela quotidiennement - l'incessante construction du site qui est toujours une origine au centre.» (Fédida, 1995, 266)

La parole, l'acte de parole est la mise en résonance de ce lieu d'origine, origine toujours à construire où les figures se déploient et façonnent alors l'histoire. Cette origine est portée en avant par la fiction sur ses origines (voir le texte de Freud, «Le roman familial des névrosés ») et elle excède toujours le sujet en raison de la dimension fantasmatique qu'elle contient et qui la maintient. L'origine est une 
tresse de temps qui s'invente une histoire et la subjectivation est liée à cette construction de temps. Le transfert est ce qui va permettre cette construction de temps car l'élaboration du récit de «fiction» est possible. Le récit reprend l'événement et lui donne lieu et mouvement là où il n'était qu'arrêt-sur-image, fixité et donc inélaborable. La parole depuis le lieu de l'infantile, recouvre les blancs d'histoire et cherche dans l'avenir les couleurs du passé. Elle est à la recherche du texte de la mémoire, ce fragment perdu dans le temps, emporté par l'événement. Aller à la recherche de ce texte perdu, c'est aussi se projeter aussi dans l'histoire et faire entrer l'événement. À défaut, le sujet restera captif des trous de mémoire, des trous dans le tissu temporel, des trous dans sa propre histoire. Le temps du travail de la cure permet d'accéder à un «présent réminiscent» (Fédida), un présent qui fait advenir l'événement pour un oubli à venir. L'oubli n'est pas le trou car l'oubli suppose une trame de temps là où le trou l'a dissous. L'effraction a défait le tissu du temps.

La cure peut devenir un appareil à relever les images, à rêver-créer dans le temps, générateur de configurations nouvelles. Le mouvement des images réarticule les strates psychiques entre elles et rend possible une écriture d'histoire. Ce temps relève de la logique du fantasme qui permet de nouer ensemble temps et image et de composer des figures en mouvement. Ce travail du temps et de la parole tressant le fantasme, ouvre des lieux de passage (au sens de Benjamin) par les figures qu'il déploie, des passages d'histoire et de mémoire.

céline masson université paris-diderot (paris-7)

3 allée jean giraudoux 78420 carrières-sur-seine france celine.masson@free.fr

\section{Notes}

1. Lettre 52 du 6-12-96. Cet exposé marque une transition théorique entre les conceptions d'un appareil psychique telles qu'il les développe dans De l'esquisse d'une psychologie scientifique (1895, paru dans le même volume cité précédemment) et celles exposées dans le chapitre 7 de L'interprétation des rêves (Die Traumdeutung, 1900). Il les reprendra dans Au-delà du principe de plaisir (1920) et dans le Bloc-notes magique (1925). L'Esquisse est une tentative d'appréhender les phénomènes psychologiques normaux et pathologiques par la description du système nerveux, système des neurones comme support de l'appareil psychique. Freud distingue perception et mémoire: il fait l'hypothèse de deux sortes de neurones: les neurones phi ou neurones de perception toujours disponibles pour recevoir des quantités venues du monde extérieur et les neurones $p s y$, neurones du souvenir, qui gardent les traces du passage des quantités. Pour une étude de l'Esquisse et des premiers développements de Freud, on se reportera au livre collectif de J.-P. Dreyfuss, J.-M. Jadin, M. Ritter, Qu'est-ce que l'inconscient? Un parcours freudien, Arcanes, Les Cahiers d'Arcanes, 1996, notamment p.57 sqq.

2. On peut distinguer la pellicule, feuillet unique, et la membrane, double feuillet. La membrane, selon ce modèle, comprend un feuillet externe assez rigide, le pare-excitation, et un feuillet interne, plus fin et plus souple mais plus fragile qui est l'enveloppe d'inscription des traces. 
3. Lorsqu'un patient s'adresse à un analyste, il lui suppose, par avance, un savoir sur ce qu'il cherche en lui-même. L'analyste est alors utilisé par l'analysant comme support d'une figure de l'Autre, d'un sujet supposé au savoir inconscient. Lacan rappelle bien qu'il ne peut y avoir de parole adressée sans cette référence à un grand Autre auquel nous nous adressons.

4. Dans son séminaire La relation d'objet, Lacan note justement à propos du texte fondamental de Freud Les trois essais sur la théorie sexuelle de 1905, que «[...] l'objet premier, précisément celui de la mère, est remémoré d'une façon qui n'a pu changer, qui est dit Freud, irréversible, de telle sorte que l'objet ne sera jamais qu'un objet retrouvé, wiedergefunden, et restera marqué du style premier de l'objet. [...] il y a donc toujours discordance de l'objet retrouvé par rapport à l'objet recherché. [...] Cette expérience fondamentale suppose qu'il y a, au cours de la période de latence, conservation de l'objet dans la mémoire, et à l'insu du sujet, c'est-à-dire transmission signifiante. Cet objet vient ensuite entrer en discordance, jouer un rôle perturbateur, dans toute relation d'objet ultérieur du sujet. C'est dans ce cadre [...] à certains temps de cette évolution, que se découvrent les fonctions proprement imaginaires.»

\section{Références}

Benjamin Walter, 1940, Sur le concept d'histoire, in Écrits français, Paris, Gallimard, Folio/essais, 1991.

Benjamin Walter, 1983, Essais, tome II, Paris, Denoël-Gonthier.

Benjamin Walter, 1989, Passages, Paris capitale du XIXe siècle, Paris, Éd. du Cerf.

Didi-Huberman Georges, 1992, Ce que nous voyons. Ce qui nous regarde, Paris, Éd. de Minuit.

Fédida Pierre, 1995, L'interlocuteur, in Le site de l'étranger - La situation psychanalytique, Paris, P.U.F.

Fédida Pierre, 1995, La régression, in Le site de l'étranger — La situation psychanalytique, Paris, P.U.F.

Fédida Pierre, 1995, La construction du cas, in Le site de l'étranger - La situation psychanalytique, Paris, P.U.F.

Ferenczi Sandor, 1974, Le problème de la fin d'analyse, in Psychanalyse IV, Euvres complètes, Paris, Payot.

Freud Sigmund, 1895, De l'esquisse d'une psychologie scientifique (Entwurf einer Psychologie), in La naissance de la psychanalyse, Paris, P.U.F., 1986.

Freud Sigmund, Lettre 52 du 6-12-96 in La naissance de la psychanalyse, Lettres à Wilhelm Fliess, Paris, P.U.F., 1986.

Freud Sigmund, 1912-1913, Totem et tabou - Interprétation par la psychanalyse de la vie sociale des peuples primitifs, G.W., IX, Paris, Gallimard, 1993.

Freud Sigmund, 1918, Extrait de l'histoire d'une névrose infantile : L'homme aux loups, in Cinq psychanalyse, Paris, P.U.F., 1990 .

Freud Sigmund, 1925, Note sur le «Bloc-notes magique», in Résultats, idées, problèmes, II (1921-1938), Paris, P.U.F., Paris, 1995.

Freud Sigmund, 1925, La négation, in Résultats, idées, problèmes, II (1921-1938), Paris, P.U.F., 1995.

Freud Sigmund, 1926, La question de l'analyse profane, Paris, Gallimard, 1985.

Goethe J-W. von, 1995, Les affinités électives, Paris-Genève, Éditions Slatkine.

Masson Céline, 2001, L'angoisse et la création - Essai sur la matière, la matière-angoisse et l'en-formation, Paris, l'Harmattan, collection dirigée par Alain Brun.

Masson Céline, 2004, La vie vaut la peine d'être visage - Essai sur le visage, avec Jean-Luc Parant, La Versanne, Encre Marine, diffusion/distribution P.U.F.

Masson Céline, 2004, Fonction de l'image dans l'appareil psychique: Construction d'un appareil optique, Toulouse, éditions Erès, collection «Actualité de la psychanalyse».

Schopenhauer Arthur, 1966, Le monde comme volonté et comme représentation, Paris, P.U.F., 1989.

Vernant Jean-Pierre, 1990, Figures, idoles, masques - Conférences, essais et leçons du Collège de France, Paris, Julliard. 